\title{
Evolution and Reversible Polarity of Multilayering at the lonic Liquid/Water Interface
}

\section{AUTHOR(S):}

Katakura, Seiji; Amano, Ken Ichi; Sakka, Tetsuo; Bu, Wei; Lin, Binhua; Schlossman, Mark L.; Nishi, Naoya

\section{CITATION:}

Katakura, Seiji ...[et al]. Evolution and Reversible Polarity of Multilayering at the Ionic Liquid/Water Interface. Journal of Physical Chemistry B 2020, 124(29): 6412-6419

\section{ISSUE DATE:}

2020-07-23

URL:

http://hdl.handle.net/2433/255281

\section{RIGHT:}

This document is the Accepted Manuscript version of a Published Work that appeared in final form in Journal of Physical Chemistry B, copyright (C) American Chemical Society after peer review and technical editing by the publisher. To access the final edited and published work see https://doi.org/10.1021/acs.jpcb.0c03711.; The full-text file will be made open to the public on 29 June 2021 in accordance with publisher's 'Terms and Conditions for Self-Archiving'.; この論文は出版 社版でありません。引用の際には出版社版をご確認ご利用ください。; This is not the published version. Please cite only the published version. 


\section{Evolution and Reversible Polarity of Multilayering at the Ionic Liquid/Water Interface}

Seiji Katakura, ${ }^{\dagger}$ Ken-ichi Amano, ${ }^{\dagger}, \ddagger$ Tetsuo Sakka, ${ }^{\dagger}$ Wei Bu, ${ }^{\top}$ Binhua Lin, "Mark L. Schlossman, ${ }^{\S}$ and Naoya Nishi*, ${ }^{*}$

$\dagger$ Department of Energy and Hydrocarbon Chemistry, Kyoto University, Kyoto 615-8510, Japan

$\ddagger$ Faculty of Agriculture, Meijo University, Nagoya, Aichi 468-8502, Japan

\ChemMatCARS, Center for Advanced Radiation Sources, University of Chicago, Chicago, IL 60637, USA

$\S$ Department of Physics, University of Illinois at Chicago, Chicago, IL 60607, USA

E-mail: nishi.naoya.7e@kyoto-u.ac.jp

Phone: +81 75-383-2491. Fax: +81 75-383-2490 


\begin{abstract}
Highly correlated positioning of ions underlies Coulomb interactions between ions and electrified interfaces within dense ionic fluids such as biological cells and ionic liquids. Recent work has shown that highly correlated ionic systems behave differently than dilute electrolyte solutions and interest is focused upon characterizing the electrical and structural properties of the dense electrical double layers (EDL) formed at internal interfaces. It has been a challenge for experiments to characterize the progressive development of the EDL on the nanoscale as the interfacial electric potential is varied over a range of positive and negative values. Here we address this challenge by measuring X-ray reflectivity from the interface between an ionic liquid (IL) and a dilute aqueous electrolyte solution over a range of interfacial potentials from $-450 \mathrm{mV}$ to $350 \mathrm{mV}$. The growth of alternately charged cation-rich and anion-rich layers was observed along with a polarity reversal of the layers as the potential changed sign. These data show that the structural development of an ionic multilayer-like EDL with increasing potential is similar to that suggested by phenomenological theories and MD simulations, although our data also reveal that the excess charge beyond the first ionic layer decays more rapidly than predicted.
\end{abstract}

\title{
Introduction
}

Ions are densely packed in ionic liquids (ILs) - which are solvent free - and other systems such as concentrated polyelectrolyte solutions and biological cells. The positions of these ions are highly correlated as the result of excluded volume interactions and strong electrostatic interactions between neighboring ions. ${ }^{1,2}$ As a result of these strong correlations, the electrical double layer (EDL) structure of ionic liquids (ILs) differs from conventional dilute electrolyte solutions. ${ }^{3,4}$ The structure of the EDL underlies electrostatic interactions between ions and electrified interfaces such as electrodes in electrochemical systems. Molecular dynamics simulations have observed the formation of EDLs consisting of ionic multilayers 
at the interface of ILs with a solid electrode. ${ }^{5-12}$ These studies revealed a gradual change from mixed cation-anion multilayers at an uncharged electrode to alternating cation-rich and anion-rich layers upon charging the electrode. ${ }^{13,14}$ Once the electrode is sufficiently charged, the first layer of ions adjacent to it overscreens the electrode, that is, the charge in this first layer exceeds the charge on the electrode. The second layer is enriched with co-ions and overscreens the first layer, but to a lesser extent. The sign of the electrode charge determines the polarity of the alternately charged ionic multilayers. Overscreening is a direct consequence of electrostatically-induced spatial correlations ${ }^{15}$ and does not appear in conventional theories of dilute electrolyte solutions, such as the Gouy-Chapman theory. ${ }^{16,17}$ Furthermore, MD ${ }^{13,14}$ and theoretical ${ }^{18,19}$ studies predict that increasing the charge on the electrode produces crowding of ions, which occurs when the electrode charge is large enough that a complete, single layer of counterions is inadequate to screen it, instead a second counterion-rich layer is required.

Experimental techniques that have been used to study the distribution of ions in the EDL structure of ILs include X-ray reflectivity (XR), ${ }^{20-28}$ neutron reflectivity, ${ }^{29-32}$ atomic force microscopy, ${ }^{33-41}$ and surface force measurements. ${ }^{42-46}$ However, results from experiments vary in several important aspects. For example, the measured thickness of the EDL ranges from a Helmholtz-like single ion dimension ${ }^{32,47}$ in some experiments to several ${ }^{20}$ and even tens ${ }^{46}$ of ions thick in others. XR studies that measure the EDL between ILs and a variety of solid electrode materials, such as $\mathrm{Au},{ }^{23} \mathrm{Si},{ }^{27}$ epitaxial graphene on $\mathrm{SiC},{ }^{24,28}$ and borondoped diamond, ${ }^{26}$ have exhibited different interfacial structures. A thick interfacial layer of several $\mathrm{nm}$ was observed at positive potentials for an IL in contact with a Si electrode, ${ }^{27}$ but it was not observed with a boron-doped diamond electrode. ${ }^{26}$ Instead, as the electric potential was varied from +1.5 to $-2.5 \mathrm{~V}$, the surface charge density decreased by only $20 \%$ of a monolayer-equivalent; the polarity of the ionic multilayers did not change, in spite of reversing the potential. Two studies have suggested a change in polarity upon reversing the potential, ${ }^{23,24}$ but the systematic growth and reversal of polarity as the interfacial potential 
is scanned from negative to positive potentials has not been observed experimentally. Here, we present such observations by utilizing X-ray reflectivity measurements to characterize the development of alternately charged ionic multilayers on the molecular-scale.

In contrast to these studies of the IL/solid electrode interface, we study the electrochemical liquid/liquid interface between IL and water $(\mathrm{W})^{48}$ using XR methods similar to those used earlier to study the electrochemical liquid/liquid interface between dilute organic and aqueous electrolyte solutions. ${ }^{49-52}$ The soft interface between an IL and an aqueous electrolyte solution may provide a useful contrast to the study of IL against hard electrode surfaces for several reasons. First, the effect of structural defects present on most solid surfaces is avoided. Second, electrostriction effects, in which ions pack closer to a solid charged electrode with an enhanced surface density as a result of an increased electrostatic attraction to the surface, ${ }^{13,14}$ should be reduced at a soft interface which can be distorted by the ions. Third, the geometric boundary condition imposed by a solid substrate induces multilayering. Many liquids, with or without ions, form molecular multilayers at the liquid/solid interface, even pure water does this, ${ }^{53}$ although it does not form multilayers at the soft water/vapor interface. ${ }^{54}$ Nevertheless, the formation of cation-anion mixed multilayers at the soft IL/vapor interface has been demonstrated by us, ${ }^{25,55,56}$ and others,. ${ }^{57,58}$ For example, our earlier XR measurements of the EDL structure at the IL/W interface for two potentials that straddle the potential of zero charge (PZC) demonstrated the presence of multilayers. ${ }^{25}$ Although multilayering appears to be an intrinsic property of ILs at all charged interfaces, the geometric constraints of a solid surface are likely to enhance it.

In the present study, we measure XR from the soft IL/W interfaces under potential control by a four-electrode system and characterize the potential dependence of the EDL structure of ILs. 


\section{Methods}

\section{Materials}

We used a hydrophobic ionic liquid, trihexyltetradecylphosphonium bis(nonafluorobutanesulfonyl)amide ${ }^{56}\left(\left[\mathrm{THTDP}^{+}\right]\left[\mathrm{C}_{4} \mathrm{C}_{4} \mathrm{~N}^{-}\right]\right)$which forms a well-defined interface with an aqueous solution of LiCl. Fig. 1(a) illustrates its structure. $\left[\mathrm{THTDP}^{+}\right]\left[\mathrm{C}_{4} \mathrm{C}_{4} \mathrm{~N}^{-}\right]$was prepared from $\left[\mathrm{THTDP}^{+}\right] \mathrm{Cl}^{-}$(Aldrich) and $\mathrm{Li}^{+}\left[\mathrm{C}_{4} \mathrm{C}_{4} \mathrm{~N}^{-}\right]$(Mitsubishi Materials Electronic Chemicals) and purified using the same method for a hydrophobic IL trioctylmethylammonium $\mathrm{C}_{4} \mathrm{C}_{4} \mathrm{~N}^{-}$ described elsewhere. ${ }^{59}$ The density of $\left[\mathrm{THTDP}^{+}\right]\left[\mathrm{C}_{4} \mathrm{C}_{4} \mathrm{~N}^{-}\right]$is $1.213 \mathrm{~g} \mathrm{~cm}^{-3}\left(=0.382 \mathrm{e}^{-3}\right)$ at $25^{\circ} \mathrm{C} .{ }^{56}$

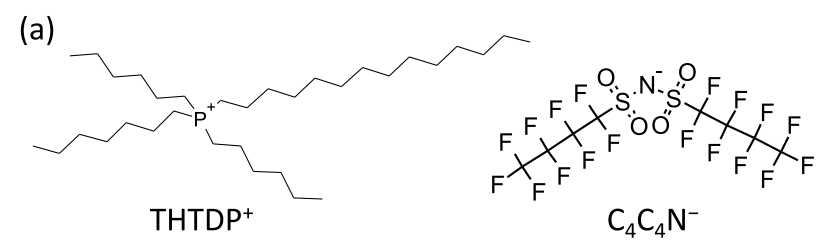

(b)
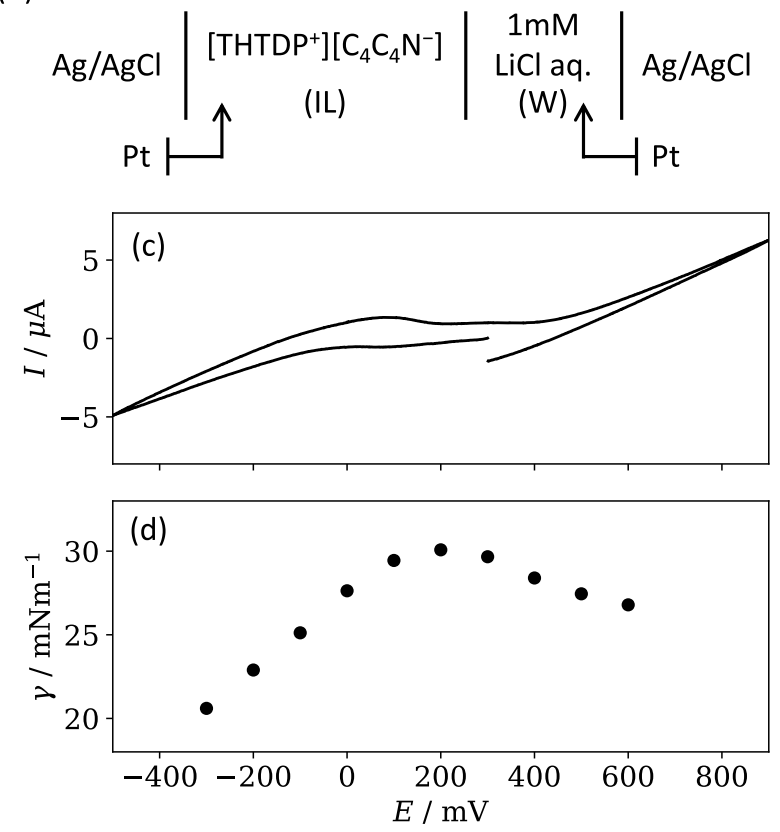

Figure 1: (a) Structure of $\left[\mathrm{THTDP}^{+}\right]\left[\mathrm{C}_{4} \mathrm{C}_{4} \mathrm{~N}^{-}\right]$. (b) Electrochemical system at the interface between $\left[\mathrm{THTDP}^{+}\right]\left[\mathrm{C}_{4} \mathrm{C}_{4} \mathrm{~N}^{-}\right]$and $1 \mathrm{mM} \mathrm{LiCl}$ aqueous solution. (c) Cyclic voltammogram at $10 \mathrm{mVs}^{-1}$. (d) Electrocapillary curve whose apex at roughly $200 \mathrm{mV}$ corresponds to the potential of zero charge. 


\section{Electrochemical system}

A four-electrode electrochemical system, shown in Fig. 1(b), is used to polarize the IL/W interface. ${ }^{48}$ Although this liquid/liquid system forms EDLs on both sides of the interface, our XR measurements are relatively insensitive to the presence of the $\mathrm{LiCl}$ EDL on the aqueous side for the following reasons. The $\mathrm{Li}^{+}$and $\mathrm{Cl}^{-}$electrolytes in water do not specifically adsorb to the IL/W interface ${ }^{60,61}$ and the low concentration of $\mathrm{LiCl}$ in the water $(1 \mathrm{mM})$ produces a low X-ray contrast of the LiCl EDL in the water.

A cyclic voltammogram of the IL/W interface is shown in Fig. 1(c), which was taken using the setup for electrocapillary measurement described below with a positive-feedback $I R$ compensation. The potential of $\mathrm{Ag} / \mathrm{AgCl}$ in the aqueous phase with respect to that in the IL phase is denoted as $E$. The potential window of the IL/W interface was estimated to span the range of -300 to $600 \mathrm{mV}$. The negative limit of the potential window corresponds to ion transfer of $\mathrm{Cl}^{-}$from $\mathrm{W}$ to $\mathrm{IL}$, and the positive limit corresponds to transfer of $\mathrm{C}_{4} \mathrm{C}_{4} \mathrm{~N}^{-}$ from IL to $\mathrm{W}$, as determined by the standard ion-transfer potentials of $\mathrm{THTDP}^{+}, \mathrm{C}_{4} \mathrm{C}_{4} \mathrm{~N}^{-}$, $\mathrm{Li}^{+}$, and $\mathrm{Cl}^{-}$in the oil-water two-phase system. ${ }^{62,63}$

\section{Electrocapillary measurement}

The interfacial tension at the IL/W interface as a function of potential (known as the electrocapillary curve) was measured. As shown in Fig. 1(b), $\mathrm{Ag} / \mathrm{AgCl}$ wires were used as the reference electrode in the water phase and the quasi-reference electrode in the IL. Platinum wires were used as the counter electrodes in the IL and water phases. The potential was controlled using a four-electrode potentiostat (Hokuto Denko, HA1010 mA 1A). A pendant drop of IL in the water phase was formed at the tip of a glass tube (inner diameter: $3 \mathrm{~mm}$ ) that was filled with the IL. After applying the potential, the drop was held for 14 minutes to complete the EDL charging relaxation. Then the picture of the droplet was taken every 5 s for $1 \mathrm{~min}$ and the outlines of the droplet were extracted. The temperature was controlled at $25^{\circ} \mathrm{C}$. The detail of the equipment and data analysis to determine the interfacial tension 
from the outline was described elsewhere. ${ }^{60,64}$

From the electrocapillary plot (Fig. 1(d)), the potential of zero charge (PZC) was estimated by the apex of the electrocapillary parabola to be roughly $200 \mathrm{mV}\left(=E_{\mathrm{PZC}}\right)$. The IL side of the interface is negatively charged when $E>E_{\mathrm{PZC}}$ and positively charged when $E<E_{\mathrm{PZC}} \cdot{ }^{60,61}$

\section{$\mathrm{X}$-ray reflectivity measurement and data analysis}

XR was performed using the liquid surface reflectometer at ChemMatCARS Sector 15 at Advanced Photon Source (APS, at Argonne National Laboratory) in the same electrochemical system as shown in Fig. 1(b). A cell made of Polyetheretherketone (PEEK) was designed to perform XR while controlling the potential at the liquid-liquid interface (Fig.2). The electrodes on the lower IL phase penetrates the cell sidewall through two sealed lateral holes. To keep the IL|W interface flat, the volume of the lower IL phase was finely adjusted using a syringe connected through another sealed lateral hole. A quartz glass frame was placed inside the cell to make a flat interface. The glass frame was pre-treated by being immersed in a saturated potassium hydroxide ethanol solution to make the surface hydrophilic and then coated with a $1 \%$ dimethyldichlorosilane-hexane solution only on the inner side surface to make it hydrophobic. This surface modification made the upper surface of the frame W-wet and the inner side surface IL-wet. ${ }^{55}$ During the measurement, the potential was controlled using the four electrode potentiostat (Solartron SI 1287).

X-ray reflectivity $R\left(q_{z}\right)$ was measured as a function of the wave vector transfer $q_{z}=$ $4 \pi / \lambda \sin \alpha$, where $\lambda$ is the wavelength of $\mathrm{X}$-ray and $\alpha$ is the incident angle of $\mathrm{X}$-ray. We used $30 \mathrm{keV}$ X-ray to mitigate the absorption of the incident and reflected X-ray passing through the upper water phase. The measured $R\left(q_{z}\right)$ were normalized by the Fresnel reflectivity $R_{\mathrm{F}}\left(q_{z}\right)$, which is a reflectivity calculated assuming an ideally flat and structureless interface between $\left[\mathrm{THTDP}^{+}\right]\left[\mathrm{C}_{4} \mathrm{C}_{4} \mathrm{~N}^{-}\right]$and water. The reflected X-ray was detected by a two-dimensional detector (Pilatus 1M). Details of the XR measurements were described 
elsewhere. ${ }^{65}$ The measurement was performed at room temperature. The electron density

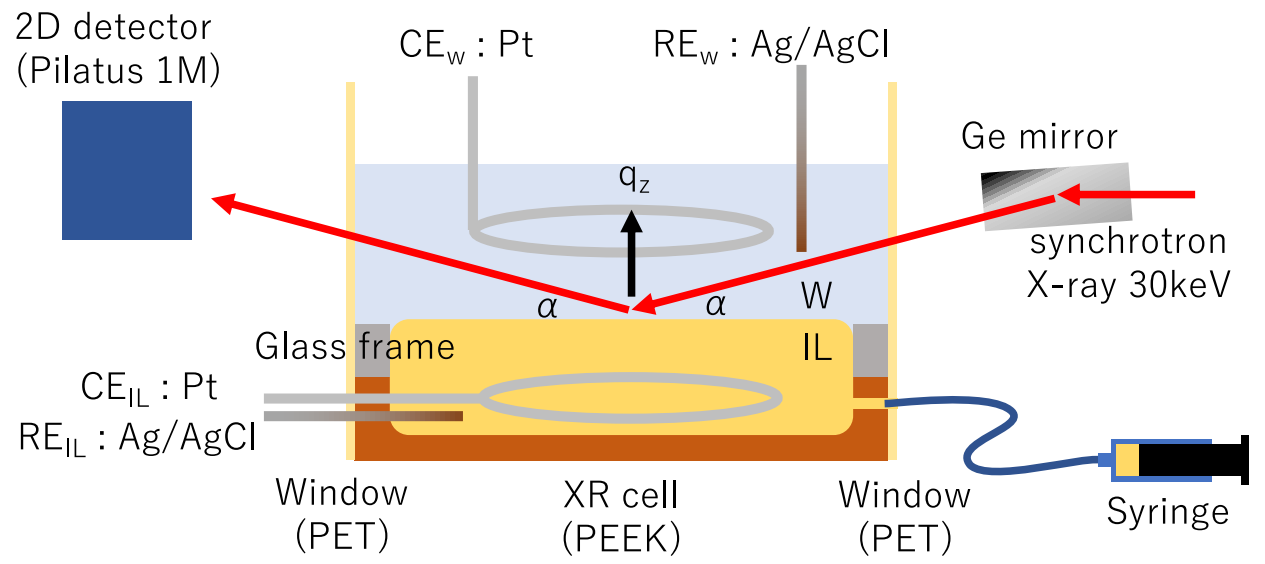

Figure 2: Schematic illustration of XR measurement and electrochemical cell.

distribution $\rho(z)$ as a function of the direction normal to the interface that reproduces experimental $R / R_{\mathrm{F}}$ was determined based on the model fitting. Note that only the EDL on the IL side of the interface is important because $1 \mathrm{mM} \mathrm{LiCl} \mathrm{aq.,} \mathrm{which} \mathrm{surely} \mathrm{forms} \mathrm{EDL}$ on the water side of the interface, is too dilute to affect the shape of the $\rho(z)$. As described above, neither $\mathrm{Li}^{+}$nor $\mathrm{Cl}^{-}$forms an adsorbed layer at the IL/W interface. ${ }^{60,61}$ Also the ionic diffuse layer on the water side has a low X-ray contrast because of such a low concentration of these relatively low-electron density elements. Therefore, by neglecting the EDL on the water side and considering ionic layers on the IL side, the $\rho(z)$ profile can be described as follows using a slab model.

$$
\rho(z)=\rho_{0}+\sum_{i=0}^{N+1}\left(\rho_{i}-\rho_{i+1}\right) \frac{1+\operatorname{erf}\left(\frac{z-z_{i}}{\sqrt{2} \sigma}\right)}{2}
$$

where $\operatorname{erf}(x)=2 / \pi \int_{0}^{x} \exp \left(-t^{2}\right) \mathrm{d} t$ is the error function. The variable $N$ is the number of interface layers, and two models with $N=1$ and 2 were used and referred to as "one slab model" and "two slab model", respectively, in the present study. In these models, $\rho(z)$ is expressed with the thickness $d_{i}=z_{i+1}-z_{i}$ and the electron density $\rho_{i}$ of the interface layer $i$, and the interface roughness $\sigma$. The electron density of the aqueous phase $(i=0)$ and 
the IL phase $(i=N+1)$ was estimated to be $\rho_{0}=\rho_{\mathrm{W}}=0.334$ and $\rho_{N+1}=\rho_{\mathrm{IL}}=0.382$ $\mathrm{e} \AA^{-3}$, respectively, from the molecular weights, densities, ${ }^{56}$ and electron numbers. $R / R_{\mathrm{F}}$ was numerically calculated from $\rho(z)$ by using Parratt method. ${ }^{66}$ The model parameters were fitted to minimize the residual sum of squares between the calculated and experimental $R / R_{\mathrm{F}}$ using a non-linear least squares method. It is meaningful to compare the roughness $\sigma$ of the slab model with the interface roughness $\sigma_{\mathrm{CWT}}$ evaluated by the capillary wave theory. The $\sigma_{\mathrm{CWT}}$ is expressed in the following equation. ${ }^{67}$

$$
\sigma_{\mathrm{CWT}}^{2}=\frac{k_{\mathrm{B}} T}{2 \pi \gamma} \ln \left(\frac{q_{\mathrm{max}}}{q_{\mathrm{res}}}\right)
$$

where $k_{\mathrm{B}}$ is the Boltzmann constant, $T$ is the absolute temperature, and $\gamma$ is the interfacial tension that is measured using the electrocapillary measurements described above. The value of $q_{\max }$ is the maximum wave number determined by the size of the molecule and is estimated as $q_{\max }=2 \pi / D_{\text {mol }} . D_{\text {mol }}=15 \AA$ was approximated from the thickness of ionic layer obtained from our previous XR study at the surface of $\left[\mathrm{THTDP}^{+}\right]\left[\mathrm{C}_{4} \mathrm{C}_{4} \mathrm{~N}^{-}\right] .{ }^{56}$ The value of $q_{\text {res }}$ is the smallest measurable wave number determined by the XR instrument and is estimated as $q_{\text {res }} \sim(h / L) q_{z}$ where $h=1.892 \mathrm{~mm}$ is the height of the detector slit and $L$ $=2.655 \mathrm{~m}$ is the distance from the center of the interface to the detector. ${ }^{65}$

\section{Model selection for electron density profile}

At first, we used the one-slab model for the electron density profile. The one-slab model has the following three parameters; electron density $\rho$ and thickness $d$ of the slab, and the interfacial roughness $\sigma$. The results of the fitting to the $R / R_{\mathrm{F}}\left(q_{z}\right)$ and the modeled electron density profiles are shown in Fig. S2, and the best-fit parameters are listed in Table S1 and shown in Fig. S1(a,c,e). The one-slab model was able to reproduce the $R / R_{\mathrm{F}}\left(q_{z}\right)$ plots at all the potentials except for 450 and $550 \mathrm{mV}$. However, the best-fit parameters were physically unreasonable. At the potentials around PZC, the thickness of the slabs ( 25 to $35 \AA$ ) was 
significantly larger than the size of ions (Fig. S1(c)). At the potentials below PZC, the roughness $\sigma$ was larger than the capillary wave roughness $\sigma_{\mathrm{CWT}}$ (Fig. S1(e,f)), which is the experimental upper limit in XR. These unreasonable parameters indicate that the one-slab model does not suit this system, although the model reproduces the shape of the electron density profile.

Then we examined other models. The distorted crystal model, ${ }^{68,69}$ which was used for the previous XR studies for the IL/solid ${ }^{20}$ and IL/air ${ }^{55}$ interfaces, also reproduced the $R / R_{\mathrm{F}}\left(q_{z}\right)$ plots. However, we decided not to use this model because it requires more fitting parameters and the $R / R_{\mathrm{F}}\left(q_{z}\right)$ plots do not always show convex behavior characteristic to this model. Next, we extended the slab model from one- to two-slab model. The extension increases the number of fitting parameters from three to five. As shown in Fig. S2 three parameters are enough to reproduce the $R / R_{\mathrm{F}}\left(q_{z}\right)$ plot. In order to prevent the overfitting, we fixed the roughness to the capillary wave roughness and used the same thickness for the first and second layers. Therefore, the fitting parameters for the two-slab model is the electron density of the first slab $\left(\rho_{1}\right)$ and second slab $\left(\rho_{2}\right)$, and the thickness of slabs $\left(d=d_{1}=d_{2}\right)$. The bestfit parameters of the two-slab model are listed in Table S2 and shown in Fig. S1(b,d). $\rho_{1}$ and $\rho_{2}$ show clear and systematic tendency to be higher and lower as potential becomes positive, crossing at $E=E_{\mathrm{pzc}}\left(\right.$ Fig. S1(b)). $d\left(=d_{1}=d_{2}\right)$ was in the order of ion diameter (Fig. S1(d)). The potential dependence (or independence) of the fitted parameters is physicochemically justifiable. The $\chi^{2}$ values for the two-slab model are similar to those for the one-slab model at most potentials with significant improvement at the two most positive potentials (Tables $\mathrm{S} 1$ and S2). Therefore we concluded that the two-slab model used here is appropriate to extract the information on the EDL structure from XR data, which is lost when using the one-slab model and also other models with more fitting parameters. 


\section{Results and discussion}

\section{X-ray reflectivity}

Measurements of normalized reflectivity $R / R_{\mathrm{F}}$ as a function of the wave vector transfer $q_{z}$ for a range of potentials are shown in Fig. 3. They provide a structural characterization of the interfacial EDL over the range of potentials $E-E_{\mathrm{PZC}}$ varying from $-450 \mathrm{mV}$ to $350 \mathrm{mV}$. Peaks develop as the potential $E-E_{\mathrm{PZC}}$ increases from 150 to $350 \mathrm{mV}$, for which the IL side of the interface is negatively charged. Since the electron density of IL $\left(\rho_{\mathrm{IL}}=0.382 \mathrm{e} \AA^{-3}\right)$ is larger than that of $\mathrm{W}\left(\rho_{\mathrm{W}}=0.334 \mathrm{e}^{-3}\right)$, the peaks indicate the formation of an interfacial layer whose $\rho$ is either higher than $\rho_{\mathrm{IL}}$ or lower than $\rho_{\mathrm{W}}$. Considering that the fluorinated anion $\mathrm{C}_{4} \mathrm{C}_{4} \mathrm{~N}^{-}$has higher $\rho$ than $\mathrm{THTDP}^{+},{ }^{25}$ and taking into account the polarity of the applied potential, the peaks reveal the formation of a high- $\rho$ layer due to an enrichment of IL anion at the interface. On the other hand, the nearly monotonic $R / R_{\mathrm{F}}$ at potentials near and below the PZC suggest a nearly monotonic variation of the electron density profile, as verified by modeling the profile to fit the reflectivity data.

We examined several models, as described above, and found that a two-slab model reproduced the $R / R_{\mathrm{F}}$ for all potentials. The two-slab model consists of two uniform bulk phases (W and IL) that sandwich two slabs, each slab is a homogeneous layer of uniform electron density, as illustrated by the dashed lines in Fig. 4. The internal interfaces in this model (W/first-slab, first-slab/second-slab, second-slab/IL) are roughened by capillary waves using a single value of roughness determined from the measured interfacial tension (Fig. 1(d)) and capillary wave theory. ${ }^{67,70}$ As will be shown, the first and second slabs describe the layering of IL cations and anions within the EDL. Although the IL cations and anions are of slightly different sizes, they are nearly the same within the resolution of these measurements. Therefore, the thicknesses of the first and second slabs were set equal in order to reduce the number of free parameters in the model. Fitted $R / R_{\mathrm{F}}$ curves and profiles of $\rho$ are shown in Figs. 3 and 4 , respectively, and the best-fit parameters, $d, \rho_{1}$, and $\rho_{2}$, are listed in Table 


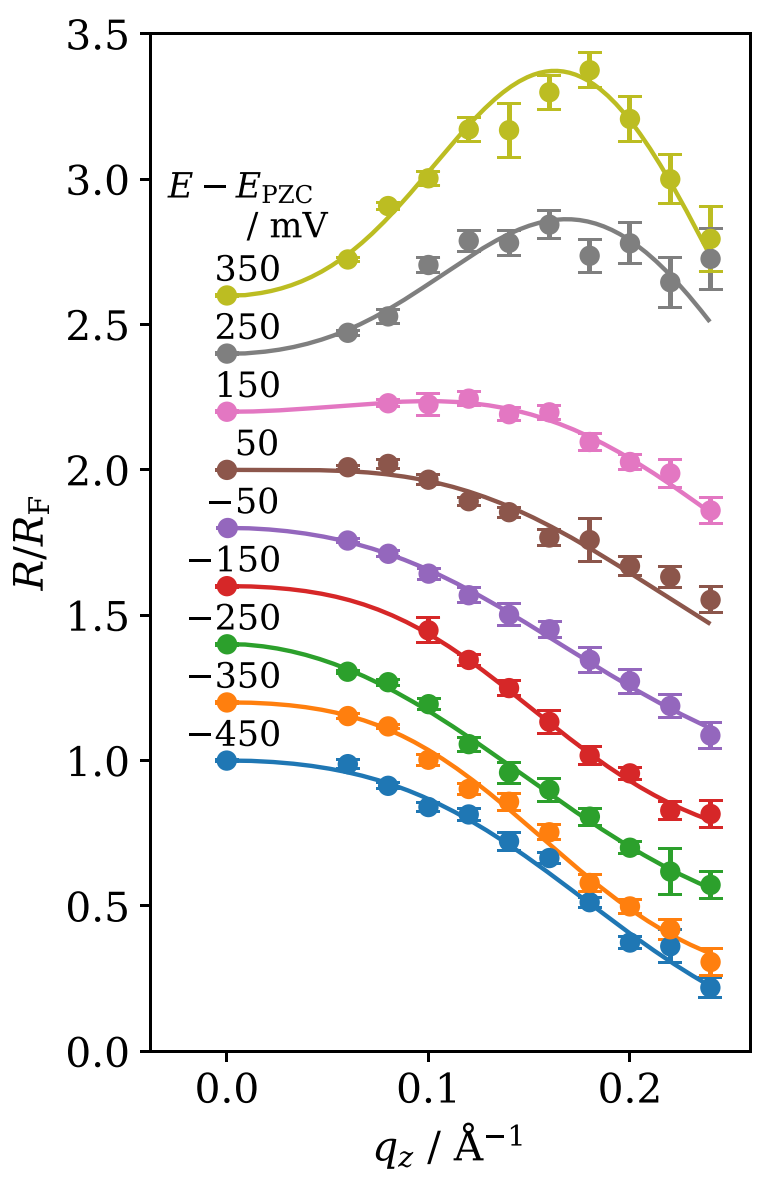

Figure 3: X-ray reflectivity $R$ normalized by the Fresnel reflectivity $R_{\mathrm{F}}$ as a function of the surface-normal wave vector transfer $q_{z}$ for several different potentials which are offset vertically for clarity. The solid lines are best-fit curves from the two-slab model described in the text. 
S2 where $d$ is the thickness and $\rho_{1}$ and $\rho_{2}$ are the electron densities of the first and second slabs.

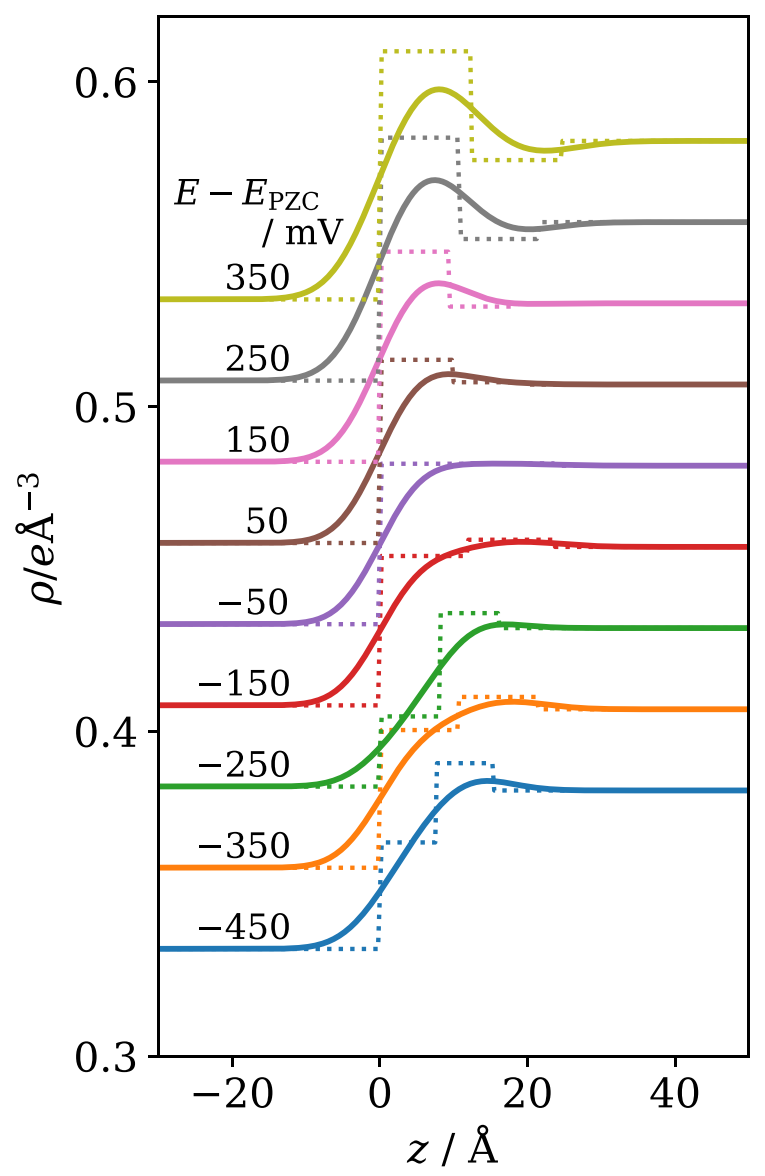

Figure 4: Electron density profiles $\rho(z)$ at the IL/W interface derived by fits to the data in Fig. 3, where $z$ is the displacement along the surface normal. Profiles at different potentials are offset for clarity. Bulk water is located at $z \leq 0$ and bulk IL at $z \geq 0$. The dotted lines represent the profiles of the two-slab model without capillary wave roughness.

Figure 4 illustrates the bilayer structure of the EDL. The fitted values of slab thickness $d$ are comparable to the layer thickness measured previously of ionic multilayers at the liquid/vapor interface of $\left[\mathrm{THTDP}^{+}\right]\left[\mathrm{C}_{4} \mathrm{C}_{4} \mathrm{~N}^{-}\right] .{ }^{56}$ At $E-E_{\mathrm{PZC}} \geq 50 \mathrm{mV}$, the first slab, which represents the ionic layer adjacent to water $(0 \leq z \leq \sim 10 \AA)$, has a higher electron density $\rho_{1}$ than the IL bulk (see Table $\mathrm{S} 2$ for numerical values). Since $\mathrm{C}_{4} \mathrm{C}_{4} \mathrm{~N}^{-}$has a higher $\rho$ than $\mathrm{THTDP}^{+}$, the first layer is enriched with anions $\mathrm{C}_{4} \mathrm{C}_{4} \mathrm{~N}^{-}$, as expected for $E>E_{\mathrm{PZC}}$. The 
electron density $\rho_{1}$ increases with increasing potential, revealing the accumulation of $\mathrm{C}_{4} \mathrm{C}_{4} \mathrm{~N}^{-}$ at the interface.

Over the same potential range $(50$ to $350 \mathrm{mV}) \rho_{2}$ is reduced to values below the IL bulk as the potential increases. This reveals cation accumulation in the second layer that accompanies anion accumulation in the first layer. These observations reveal the gradual development of alternately charged ionic multilayers in ILs as suggested previously by MD ${ }^{13,14}$ and theoretical ${ }^{18,19}$ studies. The present result confirms the generality of alternately charged ionic multilayers at IL interfaces, not just at solid electrodes, ${ }^{20,22,24}$ where the hard surface provides a geometric constraint, but also at the softer electrochemical liquid/liquid interface, which fluctuates with capillary waves.

Figure 4 reveals that the thickness of the layers increases with the potential at positive potentials, especially from 250 to $350 \mathrm{mV}$. This result of the fitting is a direct consequence of the decrease in the $q_{z}$ value of the X-ray reflectivity peak with increasing potential that is observed in Fig. 3 for these two potentials. This indicates that electrostriction effects are not substantial in these samples, if present at all, because an electrostrictive thinning of the layers would yield peaks in XR that shift to larger values of $q_{z}$ with increasing potential.

Figure 4 shows that the situation is reversed for negative potentials when $E-E_{\mathrm{PzC}} \leq$ $-150 \mathrm{mV}: \rho_{1}$ and $\rho_{2}$ are respectively lower and higher than $\rho_{\mathrm{IL}}$ (Table S2), indicating the enrichment of cations and anions, respectively, within the first and second layers. As presented above, not only the two-slab model but also the one-slab model is able to reproduce the similar shape of $\rho(z)$. However, the one-slab model requires $\sigma$ unreasonably larger than $\sigma_{\mathrm{CWT}}($ Table S2) obtained from the electrocapillary measurement and therefore is not suitable as a model to discuss the interfacial structures. The variation of best-fit parameters from two slab model (Table S2) over this range of negative potentials was less systematic than for positive potentials, primarily because of the nearly monotonic variation of the XR data. Since the electron density of the first layer is higher than water but lower than IL at these negative values of $E-E_{\mathrm{PZC}}$, the electron density profiles are also nearly monotonic 
with only a weak peak appearing in the second layer. Nevertheless, fitting results at these negative potentials indicate that $\rho_{1}<\rho_{\mathrm{IL}}<\rho_{2}$, which strongly suggests the inversion of the cation- and anion-rich bilayers triggered by changing the sign of the potential.

The presence of alternately charged ionic multilayers was observed previously by experiment, ${ }^{20,22,24}$ MD simulation, ${ }^{13,14}$ and theory ${ }^{18,19}$ at IL/solid interfaces. Alternately charged layers imply that the surface charge density of the first ionic layer exceeds that of the elec-

trode, resulting in overscreening which allows a second layer of opposite charge to form. ${ }^{18,19}$ Although simulation ${ }^{13,14}$ and theory ${ }^{18,19}$ predict that further charging of the electrode produces crowding of ions in the EDL, we did not observe crowding in this experiment. Since the XR technique would be very sensitive to the predicted structure of a crowded EDL, ${ }^{13,14}$ it is possible that higher potentials are required to achieve a crowded state.

\section{Excess of electrons}

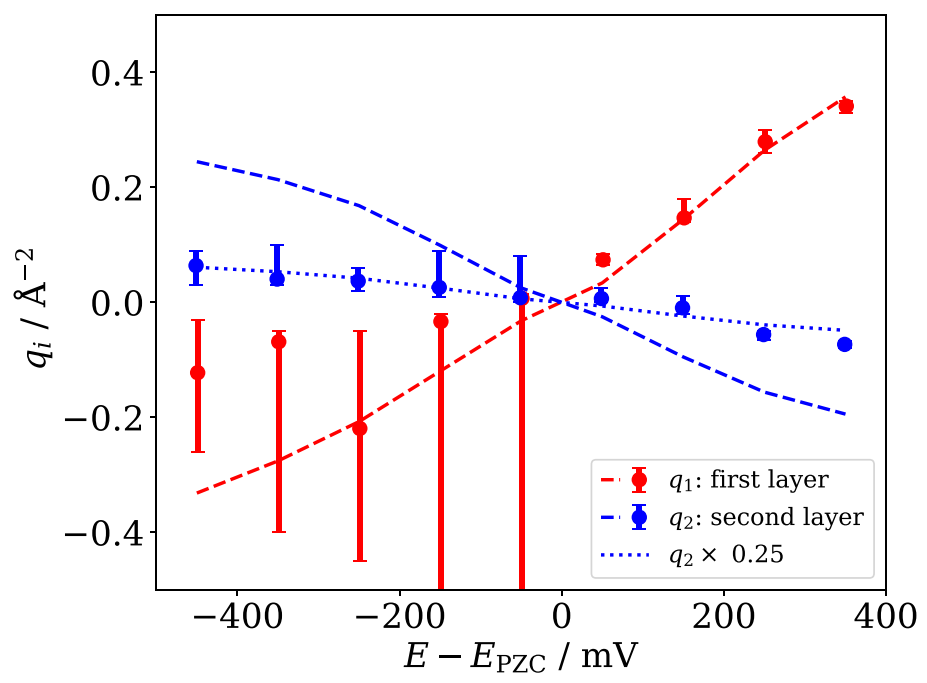

Figure 5: Electron excess in the first and second ionic layers, $q_{1}$ (red) and $q_{2}$ (blue), respectively, evaluated from the two-slab model fits to the X-ray reflectivity data. The dashed lines are $q_{1}$ (red) and $q_{2}$ (blue) from the BSK-GCS model and the dotted line is $0.25 q_{2}$ (see text and SI for details).

Further quantitative evaluation of the potential dependence of cation and anion distri- 
butions in the EDL is illustrated in Fig. 4 by the electron excess within each ionic layer, $q_{i}=\left(\rho_{i}-\rho_{\mathrm{IL}}\right) d$, where $\rho_{\mathrm{IL}}$ is the bulk electron density, $\rho_{i}$ is the layer electron density and $d$ is the layer thickness. The electron excess measures the excess surface density of electrons within a layer over that within a similar volume of bulk IL. It should be noted that $q_{i}$ is not the surface excess of ions frequently used in the electrochemical EDL analysis but still reflects the enrichment and the depletion of cations and anions at the ionic layers. Error bars in Fig. 5 were determined by $\chi^{2}$ mapping (Figs. S3-S6). With increasing potential, $q_{1}$ increased and $q_{2}$ decreased, revealing the development of alternately charged multilayer structure. Error bars are large below the PZC because the $R / R_{\mathrm{F}}$ plots in this region are relatively featureless, as discussed previously. Nevertheless, it appears that $\left|q_{1}\right|>\left|q_{2}\right|$ at all potentials, illustrating the damped oscillatory behavior of the layer-by-layer ion enrichment expected for overscreening.

To compare with the experimental electron excess $q_{i}$ we used the alternately charged multilayer model proposed by Bazant, Storey, and Kornyshev, ${ }^{18,19}$ hereafter abbreviated as the BSK model, for the EDL in the IL phase, which was combined with the Gouy-ChapmanStern (GCS) model with a $3 \AA$ thick Stern layer for the EDL in the water phase. We refer the reader to the SI for a detailed description of this BSK-GCS model. This model improves upon a model applied in a previous XR study of the IL/W interface which did not take into account the overscreening effect. ${ }^{25}$ We found that an accurate accounting of the electron excess shown in Fig. 5 required us to account for the difference in the cation and anion size that was not taken into account in the BSK model. The effect of the size asymmetry is represented by $\xi$, the volume ratio of anion to cation. Although the BSK model ${ }^{18,19}$ incorporates the Kornyshev model $^{3}$ to derive charge density distributions, we used the Oldham model, ${ }^{71}$ which treats the special case of the Kornyshev model in which compression of ions at the interface is not taken into account. Ionic compression led to a discrepancy in the $\rho$ profile from the EDL model calculation and the XR result.

The electrostatic correlation length $l_{c}$ in the BSK model was estimated to be $10 \AA$ from 
the Debye length in this IL, $\lambda_{\mathrm{D}} \sim 1.0 \AA .{ }^{18,19}$ With the ion size asymmetry $\xi$ set to be 0.75 , the $q_{1}$ curve in Fig. 5 matches the experimental values. This value of $\xi$ is comparable to a size asymmetry of 0.58 that was estimated by DFT calculations of ionic volume at the level of B3LYP/6-311G**. ${ }^{56}$ Since such calculations of a single ion in vacuum neglect the inter-ionic volume between cation and anion, ${ }^{72} \xi$ is probably underestimated in this calculation.

The $q_{2}$ curve predicted by the same parameters (blue dashed line, Fig. 5) did not agree with experimental results, suggesting that the BSK model overestimates ion enrichment in the second layer or, equivalently, underestimates the damping of the oscillatory layer-bylayer ion enrichment. Ion enrichment in the second layer is overestimated by a factor of 4 , as demonstrated by multiplying $q_{2}$ by 0.25 (Fig. 5), whose values then agree with experiment. Interestingly, we found good agreement for both $q_{1}$ and $q_{2}$ when the correlation length $l_{c}$ is taken to be smaller than the ion size. However, this may be an unphysical artifact of the model since it is unlikely that $l_{c}$ would be smaller than the ion size in the case of a globular ion like $\mathrm{THTDP}^{+}$, though packing of the more rigid $\mathrm{C}_{4} \mathrm{C}_{4} \mathrm{~N}^{-}$might lead to such an effect.

\section{Conclusions}

In summary, we investigated the potential dependence of the EDL structure at the electrified liquid/liquid interface between the ionic liquid $\left[\mathrm{THTDP}^{+}\right]\left[\mathrm{C}_{4} \mathrm{C}_{4} \mathrm{~N}^{-}\right]$and a $\mathrm{LiCl}$ aqueous solution by X-ray reflectivity. These data address the challenge posed by theory ${ }^{18,19}$ and MD simulation ${ }^{13,14}$ of measuring the gradual development of alternately charged interfacial layers as the electrical potential is varied. $\mathrm{A}_{4} \mathrm{C}_{4} \mathrm{~N}^{-}$-rich first layer and a THTDP ${ }^{+}$-rich second layer were observed at positive potentials. Although experimental uncertainties were larger at negative potentials, the formation of a $\mathrm{THTDP}^{+}$-rich first layer and $\mathrm{C}_{4} \mathrm{C}_{4} \mathrm{~N}^{-}$-rich second layer were consistent with our data at positive potentials. The present experimental results revealed the gradual development and change in polarity of alternately charged ionic multilayers upon applying a range of negative to positive potentials. Comparison of the 
excess electron surface density within each layer of the EDL with a model based upon current theory yielded good results for the density within the first layer, but poor agreement with the density in the second layer, suggesting that further development of the EDL theory for ILs is required.

\section{Acknowledgements}

The authors would like to thank D. Amoanu, C. Erol, Z. Liang, H. Miyatake, T. Arai, H. Takashina, S. Takagi, and T. Yamazawa for participation in experiments at the Advanced Photon Source (APS). NSF's ChemMatCARS Sector 15 is supported by the Divisions of Chemistry (CHE) and Materials Research (DMR), National Science Foundation, under grant number NSF/CHE-1834750. Use of the APS, an Office of Science User Facility operated for the U.S. Department of Energy (DOE) Office of Science by Argonne National Laboratory, is supported by the U.S. DOE under Contract No. DE-AC02-06CH11357. The authors would also like to acknowledge M. Ohashi and Y. Ouchi at Tokyo Institute of Technology for providing us an original version of the program code of the BSK-GCS model for calculating the potential and ionic concentration profiles. This work was partly supported by JSPS KAKENHI (No. 18K05171) and Kato Foundation for Promotion of Science.

\section{Supporting Information Available}

XR Model fitting and EDL model calculations.

\section{References}

(1) Canongia Lopes, J. N.; Pádua, A. A. Nanostructural Organization in Ionic Liquids. J. Phys. Chem. B 2006, 110, 3330-3335. 
(2) Hayes, R.; Warr, G. G.; Atkin, R. Structure and Nanostructure in Ionic Liquids. Chem. Rev. 2015, 115, 6357-6426.

(3) Kornyshev, A. A. Double-Layer in Ionic Liquids: Paradigm Change? J. Phys. Chem. B 2007, 111, 5545-5557.

(4) Fedorov, M. V.; Kornyshev, A. A. Ionic Liquids at Electrified Interfaces. Chem. Rev. 2014, 114, 2978-3036.

(5) Feng, G.; Zhang, J. S.; Qiao, R. Microstructure and Capacitance of the Electrical Double Layers at the Interface of Ionic Liquids and Planar Electrodes. J. Phys Chem C 2009, 113, 4549-4559.

(6) Kislenko, S. A.; Samoylov, I. S.; Amirov, R. H. Molecular Dynamics Simulation of the Electrochemical Interface Between a Graphite Surface and the Ionic Liquid [Bmim][PF6]. Phys. Chem. Chem. Phys. 2009, 11, 5584-5590.

(7) Wang, S.; Li, S.; Cao, Z.; Yan, T. Y. Molecular Dynamic Simulations of Ionic Liquids at Graphite Surface. J. Phys. Chem. C 2010, 114, 990-995.

(8) Vatamanu, J.; Borodin, O.; Smith, G. D. Molecular Simulations of the Electric Double Layer Structure, Differential Capacitance, and Charging Kinetics for N-methyl-npropylpyrrolidinium Bis(fluorosulfonyl)imide at Graphite Electrodes. J. Phys. Chem. B 2011, 115, 3073-3084.

(9) Merlet, C.; Salanne, M.; Rotenberg, B. New Coarse-Grained Models of Imidazolium Ionic Liquids for Bulk and Interfacial Molecular Simulations. J. Phys. Chem. C 2012, 116, 7687-7693.

(10) Haskins, J. B.; Lawson, J. W. Evaluation of Molecular Dynamics Simulation Methods for Ionic Liquid Electric Double Layers. J. Chem. Phys. 2016, 144, 184707. 
(11) Katakura, S.; Nishi, N.; Kobayashi, K.; Amano, K.; Sakka, T. An Electric Double Layer Structure and Differential Capacitance at the Electrode Interface of Tributylmethylammonium Bis(trifluoromethanesulfonyl)amide Studied Using a Molecular Dynamics Simulation. Phys. Chem. Chem. Phys. 2020, 22, 5198-5210.

(12) Katakura, S.; Nishi, N.; Kobayashi, K.; Amano, K.; Sakka, T. Effect of Switching the Length of Alkyl Chains on Electric Double Layer Structure and Differential Capacitance at the Electrode Interface of Quaternary Ammonium-Based Ionic Liquids Studied Using Molecular Dynamics Simulation. J. Phys. Chem. C 2020, 124, 7873-7883.

(13) Ivaništšev, V.; Fedorov, M. V. Interfaces between Charged Surfaces and Ionic Liquids: Insights from Molecular Simulations. Electrochem. Soc. Interface 2014, 23, 65-69.

(14) Ivaništšev, V.; Kirchner, K.; Kirchner, T.; Fedorov, M. V. Restructuring of the Electrical Double Layer in Ionic Liquids upon Charging. J. Phys.: Condens. Matter 2015, 27, 102101.

(15) Grosberg, A. Y.; Nguyen, T. T.; Shklovskii, B. I. The Physics of Charge Inversion in Chemical and Biological Systems. Rev. Mod. Phys. 2002, 74, 329-345.

(16) Chapman, D. L. A Contribution to the Theory of Electrocapillarity. Phil. Mag. 1913, 25, 475-481.

(17) Gouy, M. Sur la Constitution de la Charge Électrique à la Surface d'un Électrolyte. J. Phys. Theor. Appl. 1910, 9, 457-468.

(18) Bazant, M. Z.; Storey, B. D.; Kornyshev, A. A. Double Layer in Ionic Liquids: Overscreening Versus Crowding. Phys. Rev. Lett. 2011, 106, 046102.

(19) Bazant, M. Z.; Storey, B. D.; Kornyshev, A. A. Erratum: Double Layer in Ionic Liquids: Overscreening Versus Crowding. Phys. Rev. Lett. 2012, 109, 149903. 
(20) Mezger, M.; Schröder, H.; Reichert, H.; Schramm, S.; Okasinski, J. S.; Schröder, S.; Honkimäki, V.; Deutsch, M.; Ocko, B. M.; Ralston, J. et al. Molecular Layering of Fluorinated Ionic Liquids at a Charged Sapphire (0001) Surface. Science 2008, 322, $424-428$.

(21) Zhou, W.; Inoue, S.; Iwahashi, T.; Kanai, K.; Seki, K.; Miyamae, T.; Kim, D.; Katayama, Y.; Ouchi, Y. Double Layer Structure and Adsorption/desorption Hysteresis of Neat Ionic Liquid on Pt Electrode Surface - An In-situ IR-visible Sum-frequency Generation Spectroscopic Study. Electrochem. Commun. 2010, 12, 672-675.

(22) Zhou, H.; Rouha, M.; Feng, G.; Lee, S. S.; Docherty, H.; Fenter, P.; Cummings, P. T.; Fulvio, P. F.; Dai, S.; McDonough, J. et al. Nanoscale Perturbations of Room Temperature Ionic Liquid Structure at Charged and Uncharged Interfaces. ACS Nano 2012, $6,9818-9827$.

(23) Yamamoto, R.; Morisaki, H.; Sakata, O.; Shimotani, H.; Yuan, H.; Iwasa, Y.; Kimura, T.; Wakabayashi, Y. External Electric Field Dependence of the Structure of the Electric Double Layer at an Ionic Liquid/Au Interface. Appl. Phys. Lett. 2012, 101, 053122.

(24) Uysal, A.; Zhou, H.; Feng, G.; Lee, S. S.; Li, S.; Fenter, P.; Cummings, P. T.; Fulvio, P. F.; Dai, S.; McDonough, J. K. et al. Structural Origins of Potential Dependent Hysteresis at the Electrified Graphene/Ionic Liquid Interface. J. Phys. Chem. C 2014, $118,569-574$.

(25) Nishi, N.; Uruga, T.; Tanida, H. Potential Dependent Structure of an Ionic Liquid at Ionic Liquid/water Interface Probed by X-ray Reflectivity Measurements. J. Electroanal. Chem. 2015, 759, 129-136.

(26) Reichert, P.; Kjaer, K. S.; Brandt van Driel, T.; Mars, J.; Ochsmann, J. W.; Pontoni, D.; 
Deutsch, M.; Nielsen, M. M.; Mezger, M. Molecular Scale Structure and Dynamics at an Ionic Liquid/electrode Interface. Faraday Discuss. 2017, 206, 141-157.

(27) Chu, M.; Miller, M.; Dutta, P. Crowding and Anomalous Capacitance at an ElectrodeIonic Liquid Interface Observed Using Operando X-ray Scattering. ACS Cent. Sci. 2016, 2, 175-180.

(28) Uysal, A.; Zhou, H.; Feng, G.; Lee, S. S.; Li, S.; Cummings, P. T.; Fulvio, P. F.; Dai, S.; McDonough, J. K.; Gogotsi, Y. et al. Interfacial Ionic 'Liquids': Connecting Static and Dynamic Structures. J. Phys.: Condens. Matter 2015, 27, 032101.

(29) Lauw, Y.; Rodopoulos, T.; Gross, M.; Nelson, A.; Gardner, R.; Horne, M. D. Electrochemical Cell for Neutron Reflectometry Studies of the Structure of Ionic Liquids at Electrified Interface. Rev. Sci. Instrum. 2010, 81, 074101.

(30) Griffin, L. R.; Browning, K. L.; Clarke, S. M.; Smith, A. M.; Perkin, S.; Skoda, M. W.; Norman, S. E. Direct Measurements of Ionic Liquid Layering at a Single Mica-Liquid Interface and in Nano-Films Between Two Mica-Liquid Interfaces. Phys. Chem. Chem. Phys. 2016, 19, 297-304.

(31) Pilkington, G. A.; Harris, K.; Bergendal, E.; Reddy, A. B.; Palsson, G. K.; Vorobiev, A.; Antzutkin, O. N.; Glavatskih, S.; Rutland, M. W. Electro-Responsivity of Ionic Liquid Boundary Layers in a Polar Solvent Revealed by Neutron Reflectance. J. Chem. Phys. 2018, $148,193806$.

(32) Nishi, N.; Uchiyashiki, J.; Ikeda, Y.; Katakura, S.; Oda, T.; Hino, M.; Yamada, N. L. Potential-Dependent Structure of the Ionic Layer at the Electrode Interface of an Ionic Liquid Probed Using Neutron Reflectometry. J. Phys. Chem. C 2019, 123, 9223-9230.

(33) Horn, R. G.; Evans, D. F.; Ninham, B. W. Double-layer and Solvation Forces Measured in a Molten Salt and Its Mixtures with Water. J. Phys. Chem. 1988, 92, 3531-3537. 
(34) Liu, Y.; Zhang, Y.; Wu, G.; Hu, J. Coexistence of Liquid and Solid Phases of BmimPF6 Ionic Liquid on Mica Surfaces at Room Temperature. J Am Chem Soc 2006, 128, $7456-7457$.

(35) Atkin, R.; Warr, G. G. Structure in Confined Room-Temperature Ionic Liquids. J. Phys. Chem. C 2007, 111, 5162-5168.

(36) Bovio, S.; Podestà, A.; Lenardi, C.; Milani, P. Evidence of Extended Solidlike Layering in [Bmim][NTf2] Ionic Liquid Thin Films at Room-Temperature. J. Phys. Chem. B 2009, 113, 6600-6603.

(37) Yokota, Y.; Harada, T.; Fukui, K.-i. Direct Observation of Layered Structures at Ionic Liquid/Solid Interfaces by Using Frequency-Modulation Atomic Force Microscopy. ChemComm 2010, 46, 8627-8629.

(38) Zhang, X.; Zhong, Y.-X.; Yan, J.-W.; Su, Y.-Z.; Zhang, M.; Mao, B.-W. Probing Double Layer Structures of Au (111)-BmimPf6 Ionic Liquid Interfaces from PotentialDependent AFM Force Curves. ChemComm 2012, 48, 582-584.

(39) Li, H.; Wood, R. J.; Endres, F.; Atkin, R. Influence of Alkyl Chain Length and Anion Species on Ionic Liquid Structure at the Graphite Interface as a Function of Applied Potential. J. Phys. Condens. Matter 2014, 26, 284115.

(40) Ichii, T.; Negami, M.; Sugimura, H. Atomic-Resolution Imaging on Alkali Halide Surfaces in Viscous Ionic Liquid Using Frequency Modulation Atomic Force Microscopy. J. Phys. Chem. C 2014, 118, 26803-26807.

(41) Amano, K.; Yokota, Y.; Ichii, T.; Yoshida, N.; Nishi, N.; Katakura, S.; Imanishi, A.; Fukui, K. I.; Sakka, T. A Relationship Between the Force Curve Measured by Atomic Force Microscopy in an Ionic Liquid and Its Density Distribution on a Substrate. Phys. Chem. Chem. Phys. 2017, 19, 30504-30512. 
(42) Bou-Malham, I.; Bureau, L. Nanoconfined Ionic Liquids: Effect of Surface Charges on Flow and Molecular Layering. Soft Matter 2010, 6, 4062-4065.

(43) Ueno, K.; Kasuya, M.; Watanabe, M.; Mizukami, M.; Kurihara, K. Resonance Shear Measurement of Nanoconfined Ionic Liquids. Phys. Chem. Chem. Phys. 2010, 12, 40664071.

(44) Perkin, S. Ionic Liquids in Confined Geometries. Phys. Chem. Chem. Phys. 2012, 14, 5052-5062.

(45) Gebbie, M. A.; Dobbs, H. A.; Valtiner, M.; Israelachvili, J. N. Long-Range Electrostatic Screening in Ionic Liquids. PNAS 2015, 112, 7432-7437.

(46) Gebbie, M. A.; Smith, A. M.; Dobbs, H. A.; Lee, A. A.; Warr, G. G.; Banquy, X.; Valtiner, M.; Rutland, M. W.; Israelachvili, J. N.; Perkin, S. et al. Long Range Electrostatic Forces in Ionic Liquids. Chem Comm 2017, 53, 1214-1224.

(47) Baldelli, S. Probing Electric Fields at the Ionic Liquid-Electrode Interface Using Sum Frequency Generation Spectroscopy and Electrochemistry. J. Phys. Chem. B 2005, 109, 13049-13051.

(48) Kakiuchi, T.; Tsujioka, N.; Sueishi, K.; Nishi, N.; Yamamoto, M. Polarized Potential Window Available at the Interface Between an Aqueous Electrolyte Solution and Tetraalkylammonium Imide Salts. Electrochemistry 2004, 72, 833-835.

(49) Luo, G.; Malkova, S.; Yoon, J.; Schultz, D. G.; Lin, B.; Meron, M.; Benjamin, I.; Vanysek, P.; Schlossman, M. L. Ion Distributions Near a Liquid-Liquid Interface. Science 2006, 311, 216-218.

(50) Luo, G.; Malkova, S.; Yoon, J.; Schultz, D. G.; Lin, B.; Meron, M.; Benjamin, I.; Vanýsek, P.; Schlossman, M. L. Ion Distributions at the Nitrobenzene-Water Interface Electrified by a Common Ion. J Electroanal Chem 2006, 593, 142-158. 
(51) Laanait, N.; Mihaylov, M.; Hou, B.; Yu, H.; Vanýsek, P.; Meron, M.; Lin, B.; Benjamin, I.; Schlossman, M. L. Tuning Ion Correlations at an Electrified Soft Interface. PNAS 2012, 109, 20326.

(52) Hou, B.; Laanait, N.; Yu, H.; Bu, W.; Yoon, J.; Lin, B.; Meron, M.; Luo, G.; Vanysek, P.; Schlossman, M. L. Ion Distributions at the Water/1,2-Dichloroethane Interface: Potential of Mean Force Approach to Analyzing X-ray Reflectivity and Interfacial Tension Measurements. J. Phys. Chem. B 2013, 117, 5365-5378.

(53) Israelachvili, J. N.; Pashley, R. M. Molecular Layering of Water at Surfaces and Origin of Repulsive Hydration Forces. Nature 1983, 306, 249-250.

(54) Murphy, B. M.; Greve, M.; Runge, B.; Koops, C. T.; Elsen, A.; Stettner, J.; Seeck, O. H.; Magnussen, O. M. A Novel X-ray Diffractometer for Studies of LiquidLiquid Interfaces. J. Synchrotron Radiat. 2014, 21, 45-56.

(55) Nishi, N.; Yasui, Y.; Uruga, T.; Tanida, H.; Yamada, T.; Nakayama, S.; Matsuoka, H.; Kakiuchi, T. Ionic Multilayers at the Free Surface of an Ionic Liquid, Trioctylmethylammonium Bis(nonafluorobutanesulfonyl)amide, Probed by X-ray Reflectivity Measurements. J. Chem. Phys. 2010, 132, 164705.

(56) Nishi, N.; Uruga, T.; Tanida, H.; Kakiuchi, T. Temperature Dependence of Multilayering at the Free Surface of Ionic Liquids Probed by X-ray Reflectivity Measurements. Langmuir 2011, 27, 7531-7536.

(57) Mars, J.; Hou, B.; Weiss, H.; Li, H.; Konovalov, O.; Festersen, S.; Murphy, B. M.; Rutt, U.; Bier, M.; Mezger, M. Surface induced smectic order in ionic liquids - an X-ray reflectivity study of $[\mathrm{C} 22 \mathrm{C} 1 \mathrm{im}]^{+}[\mathrm{NTf} 2]^{-}$. Phys. Chem. Chem. Phys. 2017, 19, $26651-26661$.

(58) Pontoni, D.; Haddad, J.; Murphy, B. M.; Festersen, S.; Konovalov, O.; Ocko, B. M.; 
Deutsch, M. Surface Phases and Surface Freezing in an Ionic Liquid. The J. Phys. Chem. C 2019, 123, 3058-3066.

(59) Nishi, N.; Minami, K.; Motobayashi, K.; Osawa, M.; Sakka, T. Interfacial Structure at the Quaternary Ammonium-Based Ionic Liquids| Gold Electrode Interface Probed by Surface-Enhanced Infrared Absorption Spectroscopy: Anion Dependence of the Cationic Behavior. J. Phys. Chem. C 2017, 121, 1658-1666.

(60) Ishimatsu, R.; Shigematsu, F.; Hakuto, T.; Nishi, N.; Kakiuchi, T. Structure of the Electrical Double Layer on the Aqueous Solution Side of the Polarized Interface Between Water and a Room-Temperature Ionic Liquid, Tetrahexylammonium Bis(trifluoromethylsulfonyl)imide. Langmuir 2007, 23, 925-929.

(61) Yasui, Y.; Kitazumi, Y.; Nishi, N.; Kakiuchi, T. Analysis of Equilibrium Electrocapillary Curves at the Interface between Hydrophobic Ionic Liquid, Trioctylmethylammonium Bis(nonafluorobutanesulfonyl)amide, and Aqueous Lithium Chloride Solutions $\dagger$. J. Chem. Eng. Data 2010, 55, 4463-4466.

(62) Kakiuchi, T.; Yoshimatsu, T.; Nishi, N. New Class of Ag / AgCl Electrodes Based on Hydrophobic Ionic Liquid Saturated with AgCl. Anal. Chem. 2007, 79, 7187-7191.

(63) Nishi, N.; Murakami, H.; Yasui, Y.; Kakiuchi, T. Use of Highly Hydrophobic Ionic Liquids for Ion-Selective Electrodes of the Liquid Membrane Type. Anal. Sci. 2008, 24, 1315-1320.

(64) Kitazumi, Y.; Kakiuchi, T. Potential-Dependent Adsorption of Decylsulfate and Decylammonium Prior to the Onset of Electrochemical Instability at the 1,2Dichloroethane|Water Interface. Langmuir 2009, 25, 8062-8068.

(65) Pershan, P. S.; Schlossman, M. L. Liquid Surfaces and Interfaces: Synchrotron X-ray Methods; Cambridge University Press: Cambridge, 2012; pp 173-299. 
(66) Parratt, L. G. Surface Studies of Solids by Total Reflection of X-rays. Physical Review 1954, 95, 359-369.

(67) Braslau, A.; Pershan, P. S.; Swislow, G.; Ocko, B. M.; Als-Nielsen, J. Capillary Waves on the Surface of Simple Liquids Measured by X-ray Reflectivity. Phys. Rev. A 1988, $38,2457-2470$.

(68) Magnussen, O. M.; Ocko, B. M.; Regan, M. J.; Penanen, K.; Pershan, P. S.; Deutsch, M. X-Ray Reflectivity Measurements of Surface Layering in Liquid Mercury. Phys. Rev. Lett. 1995, 74, 4444-4447.

(69) Regan, M. J.; Kawamoto, E. H.; Lee, S.; Pershan, P. S.; Maskil, N.; Deutsch, M.; Magnussen, O. M.; Ocko, B. M.; Berman, L. E. Surface Layering in Liquid Gallium: An X-ray Reflectivity Study. Phys. Rev. Lett. 1995, 75, 2498-2501.

(70) Buff, F. P.; Lovett, R. A.; Stillinger, F. H. Interfacial Density Profile for Fluids in the Critical Region. Phys. Rev. Lett. 1965, 15, 621-623.

(71) Oldham, K. B. A Gouy-Chapman-Stern Model of the Double Layer at a (Metal)/(ionic Liquid) Interface. J Electroanal. Chem. 2008, 613, 131-138.

(72) Seki, S.; Kobayashi, T.; Kobayashi, Y.; Takei, K.; Miyashiro, H.; Hayamizu, K.; Tsuzuki, S.; Mitsugi, T.; Umebayashi, Y. Effects of Cation And Anion on Physical Properties of Room-Temperature Ionic Liquids. J. Mol. Liq. 2010, 152, 9-13. 


\section{TOC Graphic}

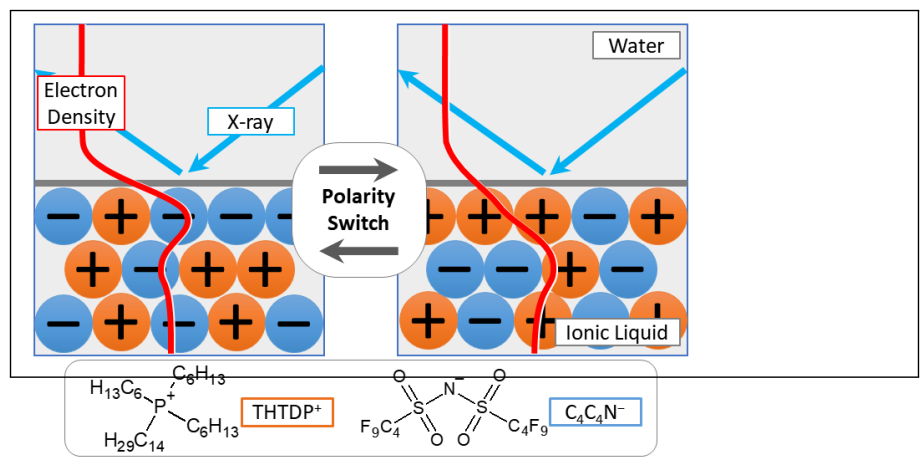

